\title{
ASYMPTOTICS OF STIRLING NUMBERS OF THE SECOND KIND
}

\author{
W. E. BLEICK AND PETER C. C. WANG ${ }^{1}$
}

\begin{abstract}
A complete asymptotic development of the Stirling numbers $S(N, K)$ of the second kind is obtained by the saddle point method previously employed by Moser and Wyman [Trans, Roy. Soc. Canad., 49 (1955), 49-54] and de Bruijn [Asymptotic methods in analysis, North-Holland, Amsterdam, 1958, pp. 102-109] for the asymptotic representation of the related Bell numbers.
\end{abstract}

1. Introduction. Hsu [1] has given the asymptotic expansion

(1) $S(N, K) \sim\left(\frac{1}{2} K^{2}\right)^{N-K}\left[1+\sum_{s=1}^{t} K^{-s} f_{s}(N-K)+O\left(K^{-t-1}\right)\right] /(N-K)$ !

for Stirling numbers $S(N, K)$ of the second kind, where $f_{s}(N-K)$ are polynomials of argument $N-K$ and $f_{s}(0)=0$. The expansion (1) is useful only for $N-K$ small, as is indicated in $\S 3$. We obtain a complete asymptotic expansion of $S(N, K)$ in powers of $(N+1)^{-1}$, using the saddle point method previously employed by Moser and Wyman [2] and de Bruijn [3] for the asymptotic representation of the related Bell numbers. Convergence is demonstrated for $K<(N+1)^{2 / 3} /\left[\pi+(N+1)^{-1 / 3}\right]$. The expansion when divergent is still useful when used as an asymptotic series.

2. Asymptotics of $S(N, K)$. A generating function for $S(N, K)$ is

$$
\left(\frac{e^{z}-1}{z}\right)^{K}=\sum_{N=K}^{\infty} \frac{K !}{N !} S(N, K) z^{N-K} \text {. }
$$

Hence the Cauchy integral formula gives

$$
S(N, K)=\frac{N !}{2 \pi i K !} \int_{C}\left(e^{z}-1\right)^{K} z^{-N-1} d z
$$

Presented to the Society, January 28, 1973; received by the editors November 6 , 1972 and, in revised form, March 12, 1973.

AMS (MOS) subject classifications (1970). Primary 41A60; Secondary 05A10.

Key words and phrases. Asymptotic expansion, Stirling number of the second kind, Bell number, saddle point method.

${ }^{1}$ This work was partially supported by the Office of Naval Research under Contract Number NR 042-286 at the Naval Postgraduate School.

(c) American Mathematical Society 1974 
where the contour $C$ encloses the origin. Equating the derivative of the integrand to zero gives the equation

$$
(t-z) e^{z-t}=t e^{-t},
$$

where $t=(N+1) / K$, for the location of the saddle point of the modulus of the integrand. The principal saddle point $z=u$ is on the positive real axis with $t-1<u<t$. The quadratic approximation to $x e^{-x}$ at $x=1$ shows that $u \approx 2 / N$ for $K=N$ and large $N$. There are other subsidiary saddle points at complex roots of (4), which we neglect in comparison with the higher saddle point at $z=u$. Since there are no other roots of (4) for $|t-z| \leqq t-u$, we may apply the Lagrange inversion formula to obtain

$$
u=t-\sum_{m=1}^{\infty} m^{m-1}\left(t e^{-t}\right)^{m} / m !
$$

convergent for $t>1$. Another form of (4) is the identity

$$
K=(N+1)\left(1-e^{-u}\right) / u
$$

needed later. Since the axis of the saddle point is perpendicular to the real axis, the part of the contour $C$ descending from $z=u$ is taken as the line $z=u+i y,|y|<\infty$, parallel to the imaginary axis. The modulus of the integrand in (3) is maximal at $z=u$ on this path, since both $\left(e^{z}-1\right)^{K}$ and $z^{-N-1}$ have this property. The closed contour $C$ is completed by a half circle of infinite radius enclosing the origin. The contribution to the integral (3) on this semicircular path is zero since $N>0$. The integral in (3) then becomes

where

$$
i\left(e^{u}-1\right)^{K} u^{-N-1} \int_{-\infty}^{\infty} \exp \psi(u+i y) d y
$$

$$
\psi(z)=K \ln \left[\left(e^{z}-1\right) /\left(e^{u}-1\right)\right]-(N+1) \ln (z / u) .
$$

The contribution of the various parts of the $z=u+i y$ path to the integral must now be studied. As $|\exp \psi(z)|=\exp \operatorname{Re} \psi(z)$ we have to study

$$
\begin{aligned}
\operatorname{Re} \psi(u+i y)= & K \ln \left[\left(e^{2 u}-2 e^{u} \cos y+1\right)^{1 / 2} /\left(e^{u}-1\right)\right] \\
& -(N+1) \ln \left(1+y^{2} u^{-2}\right)^{1 / 2}
\end{aligned}
$$

We shall show that we can restrict ourselves essentially to the interval $|y|<\pi$. Since $1+y^{2} u^{-2} \geqq 1+\pi(2 y-\pi) u^{-2}$ for $y \geqq \pi$ we have

$$
\left(e^{u}-1\right)^{K} u^{-N-1}\left|\int_{\pi}^{\infty} \exp \psi(u+i y) d y\right|<\frac{u^{1-N}\left(e^{u}+1\right)^{K}}{\pi(N-1)\left(1+\pi^{2} u^{-2}\right)^{N / 2-1 / 2}}
$$


which is of $O\left(N^{-N} e^{N}\right)$ for $K$ small and of $O\left(2^{N} / \pi^{N} N\right)$ for $K$ large. Since $\operatorname{Re} \psi(u+i y)$ is even, the part of the integral (7) for $|y|>\pi$ tends toward zero as $N \rightarrow \infty$. We now direct our attention to the interval $|y|<\pi$ where the saddle point at $y=0$ gives the main contribution. The Taylor expansion of $\psi(u+i y)$, convergent for $|y|<u$, is

$$
\begin{aligned}
\psi= & -\frac{N+1}{2 u}\left(\frac{1}{u}-\frac{1}{e^{u}-1}\right) y^{2} \\
& +(N+1) \sum_{j=1}^{\infty} \frac{(i y)^{j+2}}{(j+2) !}\left(\frac{d}{d z}\right)^{j+1}\left[\frac{1-e^{-u}}{u\left(e^{z}-1\right)}-\frac{1}{z}\right]_{z=u}
\end{aligned}
$$

where the identity (6) has been used. We now make the substitutions

$$
w=[(N+1) / 2]^{1 / 2}\left[1-u /\left(e^{u}-1\right)\right]^{1 / 2} y / u
$$

and

$$
a_{j}=\frac{(i w u)^{j+2}(d / d z)^{j+1}\left[\left(1-e^{-u}\right) / u\left(l^{z}-1\right)-1 / z\right]_{z=u}}{(j+2) !\left[\frac{1}{2}-\frac{1}{2} u /\left(e^{u}-1\right)\right]^{j / 2+1}}
$$

to obtain

$$
S(N, K)=B \int_{-\infty}^{\infty} \exp \left\{-w^{2}+f\left[(N+1)^{-1 / 2}\right]\right\} d w
$$

where

$$
B=N !\left(e^{u}-1\right)^{K} / \pi(2(N+1))^{1 / 2} K ! u^{N}(1+u /(1-\exp u))^{1 / 2}
$$

and $f$ is the analytic continuation of

$$
f\left[(N+1)^{-1 / 2}\right]=\sum_{j=1}^{\infty} a_{j}(N+1)^{-j / 2} .
$$

To find an upper bound to $\left|a_{j}\right|$ we note that $\left(e^{z}-1\right)^{-1}=\sum_{k=1}^{\infty} e^{-k z}$ for, $\operatorname{Re} z>0$. Then

$$
(d / d u)^{n}\left(e^{u}-1\right)^{-1}=(-1)^{n} \sum_{x=0}^{\infty} g(x)
$$

where $g(x)=x^{n} e^{-u x}$. On using the Euler-Maclaurin sum formula [5] we find

$$
\sum_{x=0}^{\infty} g(x)=\frac{n !}{u^{n+1}}+R_{n}
$$

The remainder $R_{n}=\int_{0}^{\infty}\left(x-[x]-\frac{1}{2}\right) g^{\prime}(x) d x$ may be evaluated by a Laplace transform [6] to be

$$
R_{n}=(-1)^{n-1}\left[n F^{(n-1)}(u)+u F^{(n)}(u)\right]
$$


where $F(u)=u^{-2}-\frac{1}{2} u^{-1} \operatorname{coth} \frac{1}{2} u$. We conclude that $\left|R_{n}\right| \ll n ! / u^{n+1}$ for small $u$ and tends to zero for large $u$. Since $\left(1-e^{-u}\right) / u$ is less than unity we have

$$
\left|a_{j}\right|<\sigma^{j+2} \mid j
$$

where

$$
\sigma=w 2^{1 / 2} /\left(1-u /\left(e^{u}-1\right)\right)^{1 / 2}=y(N+1)^{1 / 2} / u .
$$

Remembering that we need not integrate (7) beyond $|y|=\pi$ for large $N$, we see by (15) and (16) that the series (14) is convergent for $\pi / u<1$. We now expand $\exp f\left[(N+1)^{-1 / 2}\right]$ in a Taylor series of the form

$$
\exp f\left[(N+1)^{-1 / 2}\right]=\sum_{j=0}^{\infty} b_{j}(N+1)^{-j / 2}
$$

where $b_{0}=1$ and $b_{j}$ are polynomials in $w$ of the degree and parity of $3 j$. By a lemma of Moser and Wyman [4]

$$
\left|b_{j}\right| \leqq \sigma^{j+2}\left(1+\sigma^{2}\right)^{j-1}
$$

Using (17) we may write (12) in the form

$$
S(N, K)=B\left[\sum_{j=0}^{s-1}(N+1)^{-j} \int_{-\infty}^{\infty} e^{-w^{2}} b_{2 j} d w+R_{s}\right] .
$$

The absolute value of the remainder $R_{s}$ is found from (18) to be

$$
\left|R_{s}\right| \leqq(N+1)^{-s} \int_{-\infty}^{\infty} e^{-w^{2}} P_{s}(|w|) d w / M
$$

where $P_{s}(|w|)$ is a polynomial in $|w|$ and

$$
M=1-\sigma^{2}\left(1+\sigma^{2}\right)^{2} /(N+1) .
$$

On limiting the integration in (7) to $|y|<\pi$ we see that the remainder $R_{s}$ exists if

$$
(\pi / u)\left[1+(N+1)(\pi / u)^{2}\right]<1 .
$$

Since $u+1>(N+1) / K$ convergence occurs for

$$
K<(N+1)^{2 / 3} /\left[\pi+(N+1)^{-1 / 3}\right]
$$

approximately. For these values of $K$ we conclude that

(20) $S(N, K) \sim B\left\{\sum_{j=0}^{s-1}(N+1)^{-j} \int_{-\infty}^{\infty} e^{-w^{2}} b_{2 j} d w+O\left[(N+1)^{-s}\right]\right\}$. 
The first two terms of (20) have been calculated to be

$$
\begin{aligned}
S(N, K) \sim & \frac{N !\left(e^{u}-1\right)^{K}}{(2 \pi(N+1))^{1 / 2} K ! u^{N}(1-G)^{1 / 2}} \\
& \cdot\left[1-\frac{2+18 G-20 G^{2}\left(e^{u}+1\right)}{24(N+1)(1-G)^{3}}\right. \\
& \left.\quad+\frac{3 G^{3}\left(e^{2 u}+4 e^{u}+1\right)+2 G^{4}\left(e^{2 u}-e^{u}+1\right)}{24(N+1)(1-G)^{3}}\right]
\end{aligned}
$$

where by [5]

$$
G=u /\left(e^{u}-1\right)=1-\frac{1}{2} u+\sum_{k=1}^{\infty} B_{2 k} u^{2 k} /(2 k) !
$$

The bracketed expression in (21), argumented by an additional inverse power of $N+1$, is approximated by

for small $u$ and by

$$
1-\frac{1}{6 u(N+1)}+\frac{1}{72 u^{2}(N+1)^{2}}
$$

$$
1-\frac{1}{12(N+1)}+\frac{1}{288(N+1)^{2}}
$$

for large $u$. These are the leading terms of an alternating asymptotic series.

3. Numerical example. The 6-significant-figure Table 1 compares the

\begin{tabular}{|c|c|c|c|c|}
\hline $\boldsymbol{K}$ & $\begin{array}{l}S(100, K) \\
\text { Exact }\end{array}$ & $\begin{array}{c}S(100, K) \\
1 \text { term of }(20)\end{array}$ & $\begin{array}{c}S(100, K) \\
2 \text { terms of }(20)\end{array}$ & $\begin{array}{c}S(100, K) \\
4 \text { terms of }(1)\end{array}$ \\
\hline 2 & $6.3382510^{20}$ & $6.34348 \quad 10^{20}$ & $6.3382510^{20}$ & $1.8118610^{-110}$ \\
\hline 25 & $2.5832010^{114}$ & $2.5849610^{114}$ & $2.58321 \quad 10^{114}$ & $2.9469610^{83}$ \\
\hline 50 & $4.3098310^{101}$ & $4.3090010^{101}$ & $4.3097710^{101}$ & $1.5152910^{04}$ \\
\hline 75 & $1.8258410^{63}$ & $1.82671 \quad 10^{63}$ & $1.8257910^{63}$ & $5.3262610^{62}$ \\
\hline 99 & $4.9500010^{3}$ & $5.1419910^{3}$ & $4.9445110^{3}$ & $4.9500010^{8}$ \\
\hline
\end{tabular}
exact values of $S(100, K)$ with the values computed from (20) and (1) for several $K$. The excellent results obtained from (20) for values of $K$ outside the interval of convergence show that the expansion gives useful results when used as an asymptotic series.

TABLE 1

\section{REFERENCES}

1. L. C. Hsu, Note on an asymptotic expansion of the nth difference of zero, Ann. Math. Statist. 19 (1948), 273-277. MR 9, 578. 
2. Leo Moser and Max Wyman, An asymptotic formula for the Bell numbers, Trans. Roy. Soc. Canad. Sect. III (3) 49 (1955), 49-54. MR 17, 1201.

3. N. G. de Bruijn, Asymptotic methods in analysis, Bibliotheca Mathematica, vol. 4, North-Holland, Amsterdam; Groningen and Interscience, New York, 1958, pp. 102-109. MR 20 \#6003.

4. Leo Moser and Max Wyman, On solutions of $X^{d}=1$ in symmetric groups, Canad. J. Math. 7 (1955), 159-168. MR 16, 904.

5. Konrad Knopp, Theory and applications of infinite series, Blackie and Son, London, 1928, pp. 523-528.

6. G. E. Roberts and H. Kaufman, Table of Laplace transforms, Saunders, Philadelphia, 1966, p. 137. MRं 32 \#8050.

Department of Mathematics, Naval Postgraduate School, Monterey, CaliFORNIA 93940 